\title{
Standardised cement augmentation of the PFNA using a perforated blade: A new technique and preliminary clinical results. A prospective multicentre trial
}

\author{
C. Kammerlander ${ }^{\mathrm{a}, *}$, F. Gebhard ${ }^{\mathrm{b}}$, C. Meier ${ }^{\mathrm{c}}$, A. Lenich $^{\mathrm{d}}$, W. Linhart ${ }^{\mathrm{e}}$, B. Clasbrummel $^{\mathrm{f}}$, \\ T. Neubauer-Gartzke ${ }^{g}$, M. Garcia-Alonso ${ }^{\text {h}}$, T. Pavelka ${ }^{\mathrm{i}}$, M. Blauth $^{\mathrm{a}}$ \\ a Department of Trauma Surgery and Sports Medicine, Medical University of Innsbruck, Innsbruck, Austria \\ ${ }^{\mathrm{b}}$ Department of Traumatology, Hand-, Plastic-, and Reconstructive Surgery. Center of Surgery, Center of Musculoskeletal Research, University of Ulm, Ulm, Germany \\ ${ }^{\mathrm{c}}$ Department of Traumatology, Stadtspital Waid, Zürich, Switzerland \\ ${ }^{\mathrm{d}}$ Department of Traumatology, Klinikum Rechts der Isar, Technical University of Munich, Munich, Germany \\ e Department of Orthopedics and Trauma Surgery, SLK Kliniken Heilbronn, Heilbronn, Germany \\ ${ }^{\mathrm{f}}$ Department of Trauma Surgery, Zollernalb Klinikum, Balingen, Germany \\ ${ }^{\mathrm{g}}$ Department of Trauma Surgery, Kliniken Nordoberpfalz, Weiden, Germany \\ ${ }^{\mathrm{h}}$ Department of Orthopedics, Hospital Universitarion Rio Hortega, Vallodolid, Spain \\ i Department of Trauma Surgery, Medical University of Plzen, Alej Svobody 80, CZ-30460 Plzen, Czech Republic
}

A R T I C L E I N F O

Article history:

Accepted 11 July 2011

\section{Keywords:}

Hip fracture

Augmentation

PMMA

Cement leakage

PFNA

Cut-out

Cement augmentation

Osteoporosis

Cortical thickness index

Cement distribution

\section{S U M M A R Y}

Pertrochanteric fractures are a rising major health-care problem in the elderly and their operative stabilisation techniques are still under discussion. Furthermore, complications like cut-out are reported to be high and implant failure often is associated with poor bone quality. The PFNA ${ }^{\mathbb{R}}$ with perforated blade offers a possibility for standardised cement augmentation using a polymethylmethacrylate (PMMA) cement which is injected through the perforated blade to enlarge the load-bearing surface and to diminish the stresses on the trabecular bone. The current prospective multicentre study was undertaken to evaluate the technical performance and the early clinical results of this new device.

In nine European clinics, 59 patients ( 45 female, mean age 84.5 years) suffering from an osteoporotic pertrochanteric fracture (Arbeitsgemeinschaft für Osteosynthesefragen, AO-31) were treated with the augmented PFNA ${ }^{\circledR}$. Primary objectives were assessment of operative and postoperative complications, whereas activities of daily living, pain, mobility and radiologic parameters, such as cement distribution around the blade and the cortical thickness index, were secondary objectives.

The mean follow-up time was 4 months where we observed callus healing in all cases. The surgical complication rate was $3.4 \%$ with no complication related to the cement augmentation. More than onehalf of the patients reached their prefracture mobility level within the study period. A mean volume of $4.2 \mathrm{ml}$ of cement was injected. We did not find any cut-out, cut through, unexpected blade migration, implant loosening or implant breakage within the study period.

Our findings lead us to conclude that the standardised cement augmentation using the perforated blade for pertrochanteric fracture fixation enhances the implant anchorage within the head-neck fragment and leads to good functional results.

(c) 2011 Elsevier Ltd. All rights reserved.
Pertrochanteric fractures are a rising major health-care problem in the elderly and their operative stabilisation techniques are still under discussion. Whereas the dynamic hip screw is the standard fixation method for stable A1 fractures, ${ }^{1}$ there is a trend to use intramedullary implants to fix unstable A2 and A3 fractures. $^{2-7}$

* Corresponding author at: Department of Trauma Surgery and Sports Medicine, Medical University of Innsbruck, Anichstrasse 35, A-6020 Innsbruck, Austria. Tel.: +4351250480882.

E-mail address: christian.kammerlander@uki.at (C. Kammerlander).
Recent studies revealed that the PFNA is a very effective implant for the fixation of pertrochanteric femoral fractures., ${ }^{7,8}$ Bladerelated complications with the PFNA, such as cut-out, and cut through with either medial blade migration into the hip joint or lateral blade migration are reported from $0.6 \%^{9}$ over $2.6 \%$ up to $3.6 \%{ }^{7}$ However, several other implants which use a screw design for the cephalic part of the implant lead to cut-out rates up to $16 \%^{2,10,11}$

A crucial point is that these catastrophic failures mainly take place in severe osteoporotic bone. ${ }^{12}$ However, the above-mentioned studies ${ }^{7-9}$ do not only include fragility fractures and therefore the cut-out rate in the elderly may be even higher.

0020-1383/\$ - see front matter (c) 2011 Elsevier Ltd. All rights reserved. doi:10.1016/j.injury.2011.07.010 
Several biomechanical investigations on human cadaveric proximal femoral fractures showed a higher cut-out resistance when the device was augmented with polymethylmethacrylate (PMMA) cement. ${ }^{13,14}$

Augmentation of fixation devices enlarges the bone-implant interface and leads to a higher stability also in clinical use. ${ }^{10,15-21} \mathrm{~A}$ main problem regarding augmentation at the hip was the lack of a device for controlled cement placement around the implant. ${ }^{22}$ Furthermore, the discussion about cement-related disturbance of the bone metabolism is ongoing. ${ }^{13,23,24}$ However, several studies report no damage to the cartilage or the bone itself by cement augmentation. ${ }^{25-28}$ The current prospective multicentre study was undertaken to evaluate the early clinical results and the technical performance of the new standardised augmentation for the PFNA blade. To our knowledge, this is the first report on this device.

\section{Materials and methods}

The study was performed at nine orthopaedic departments between October 2009 and July 2010. The inclusion criteria were as follows: pertrochanteric fracture (Arbeitsgemeinschaft für Osteosynthesefragen/Orthopaedic Trauma Association, AO/OTA 31A), age 65 years and above, low-energy trauma and signed informed consent. Patients with a pathological fracture, any patients with active malignancy, organ transplantation or infection were excluded. The ethical commission approved the study and every single patient signed the informed consent form. A total number of 78 patients have been included. Nine (11.6\%) cases were lost to follow-up due to concurrent indisposition or weakness, which made an additional evaluation impossible. Ten patients died for reasons not related to the surgical procedure. The remaining 59 patients were followed up according to the study protocol.

\section{Operative technique}

The Proximal Femur Nail Antirotation (PFNA, Synthes ${ }^{\circledR}$, Switzerland) is available in four sizes (standard, small, XS and long) with right and left options for the long nail. The perforated blade (Fig. 1) has three holes at every deepening of the helix. A side-opening cannula (Fig. 2) allows for deliberate cement placement through the holes of the blade. Augmentation is performed with a high-viscosity PMMA cement (Traumacem, Synthes ${ }^{\circledR}$, Switzerland). Fracture reduction and implantation of the PFNA are performed as previously reported ${ }^{7,9}$ and, instead of the standard blade, the perforated blade is used. It is of utmost importance to not perforate the femoral head when drilling the $k$ wire to determine the position of the helical blade. At the end of the usual procedure and before augmentation, a perforation in the joint has to be excluded to avoid a leakage. Therefore, the sideopening cannula is inserted into the PFNA blade and some customary water-soluble contrast medium is applied with a usual syringe. If there is no contrast fluid leakage into the hip joint detected, the procedure is to be continued. Fig. 3 shows the typical

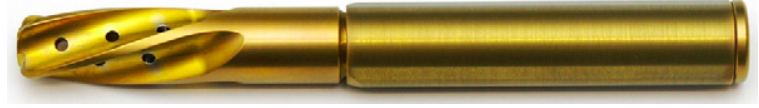

Fig. 1. The perforated blade.

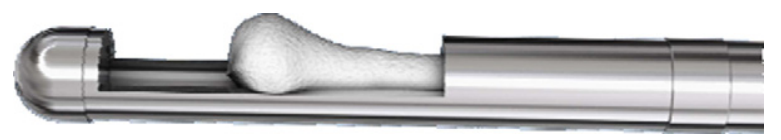

Fig. 2. The side opening cannula which is used to inject the cement.

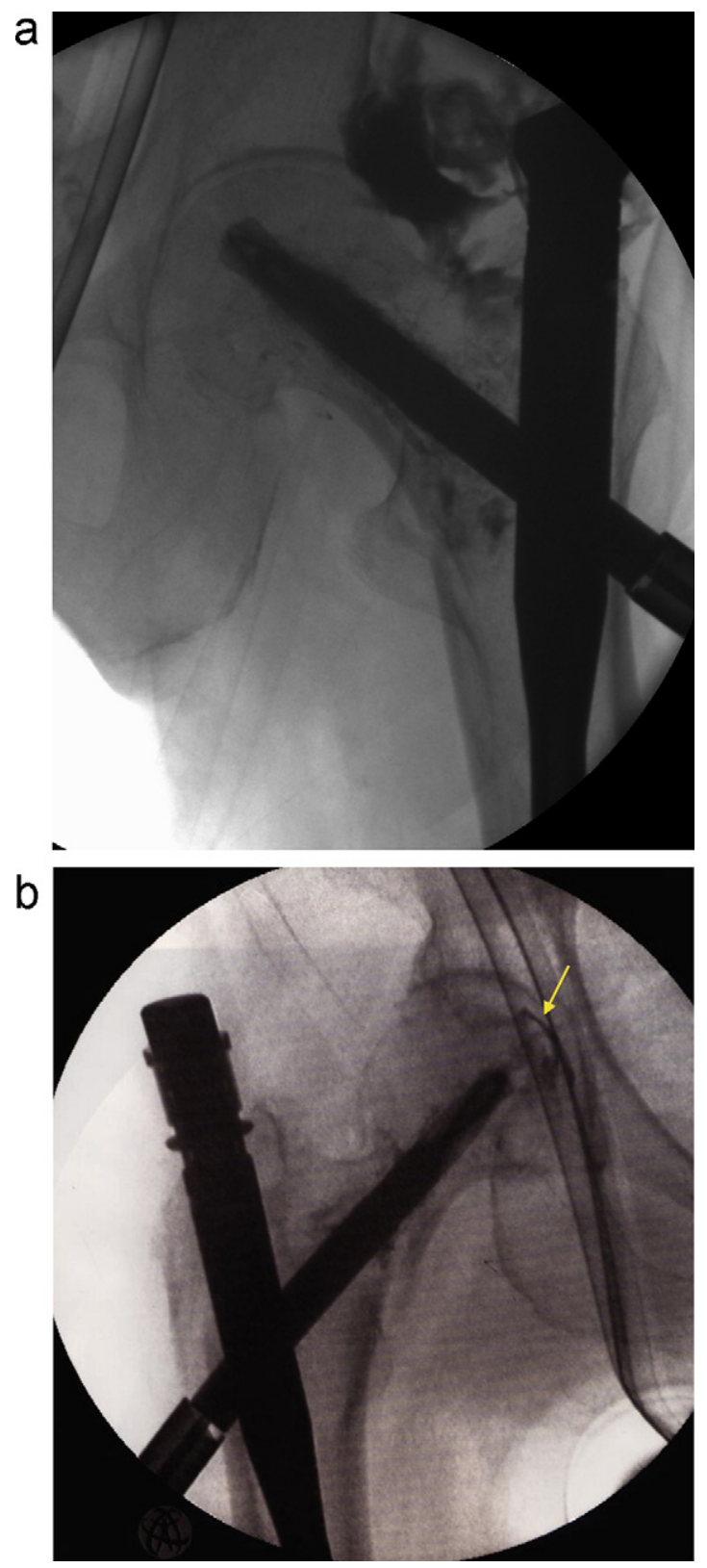

Fig. 3. Image intensifier pics with typical contrast medium distribution (a) and a case of leakage of contrast medium into the hip joint (b) which consequently prohibits cement augmentation.

distribution of the contrast medium (a) and a case of leakage (b). The cement is mixed as suggested by the manufacturer and filled into syringes with a standardised set. The side-opening cannula has to be primed with $3 \mathrm{ml}$ of cement. The syringes are then attached to the side-opening cannula and the cement is injected under fluoroscopic control. During injection, the side-opening cannula can be rotated to place the cement in different directions. After finishing the injection, the cannula has to be removed. The augmentation process lasts around $10-15 \mathrm{~min}$. Fig. 4 shows a representative case of standardised cement augmentation of the perforated PFNA blade in a 87-year-old lady with an unstable pertrochanteric fracture.

\section{Outcome parameters}

The WHO Performance Score ${ }^{29}$ was used to measure the quality of life before and after the fracture. It consists of five levels in which 
a

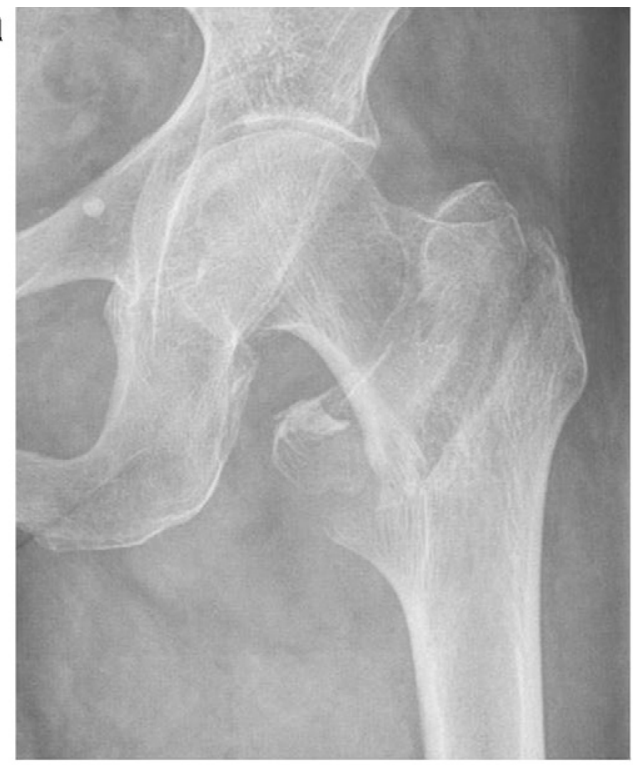

b

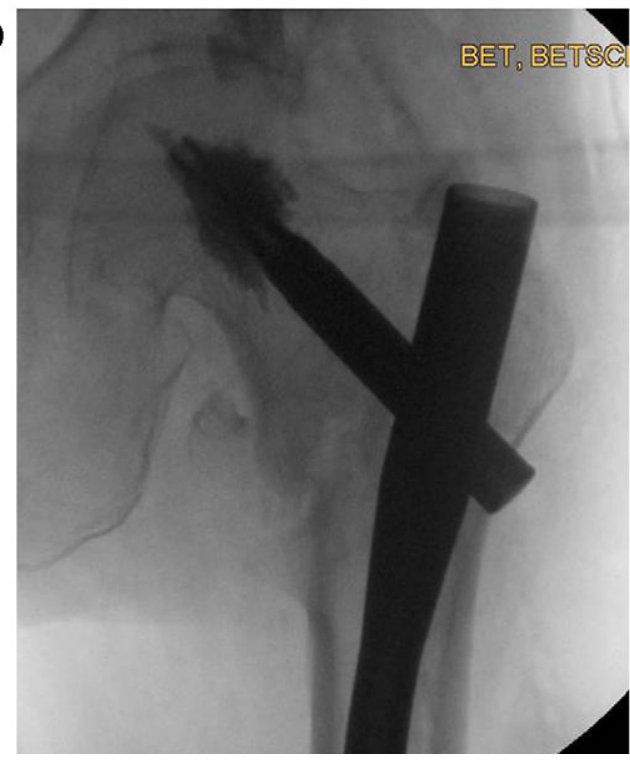

C

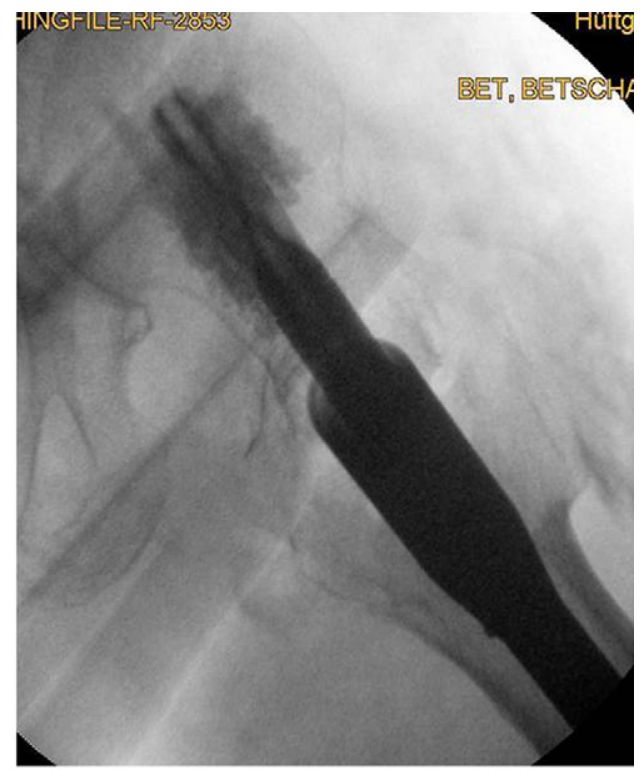

d

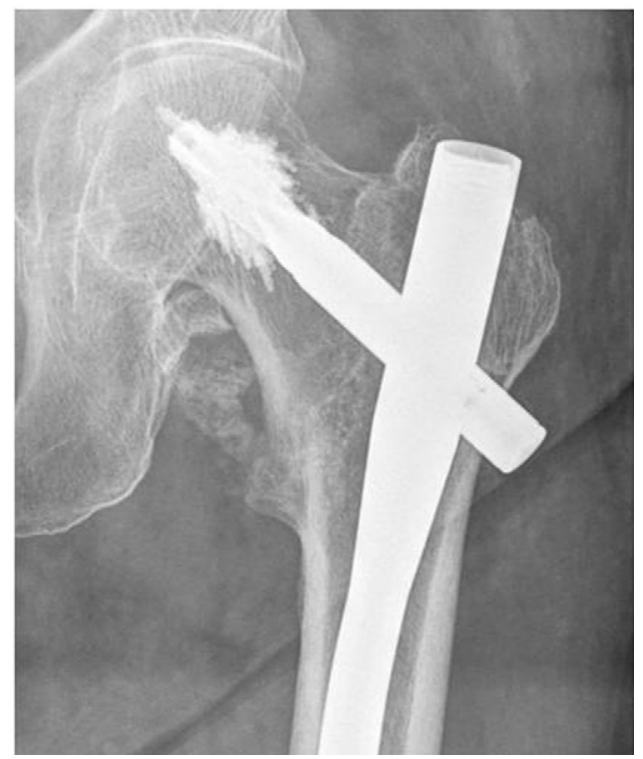

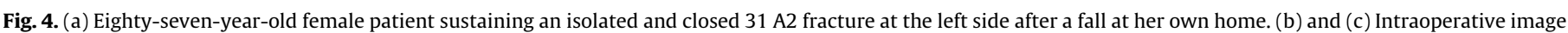

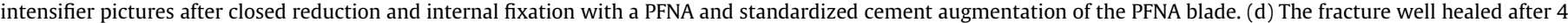
months where the patient is walking with her walking aid independently and pain-free.

0 means full activity without restriction and 4 means completely disabled and totally confined to bed or chair.

The Parker Mobility Score ${ }^{30}$ was used to assess the walking ability before the accident and at follow-up. The particular capability to walk inside, walk outside and having social contact is evaluated in four levels with "no difficulty," "alone," "with help from another person" and "not at all." A maximum of 9 points means unlimited walking ability. In addition, the use of a walking aid was documented for every patient before and after the accident.

Pain was assessed using the visual analogue scale (VAS), as previously described ${ }^{31}$ and widely known. The VAS was found to have good measurement properties assessing pain in hip fracture patients. $^{32}$

On the preoperative X-rays, the fracture pattern and the cortical thickness index ${ }^{33}$ were assessed. The cortical thickness index shows a significant positive correlation with the T-Score of the femoral neck ${ }^{34}$ and was therefore used to classify the local bone quality in our study population. A cortical thickness index lower than 0.40 (lateral film) and 0.50 (anterioposterior (ap) film) has been described as a threshold for osteoporosis where all measured femora had a local bone mineral density lower than 2.5 standard deviations below the peak bone mass, which is the World Health Organization (WHO) definition of osteoporosis. ${ }^{34}$

On the postoperative X-rays, we evaluated the quality of fracture reduction as anatomic (no displacement), near-anatomic ( $<3 \mathrm{~mm}$ displacement or $5-10^{\circ}$ varus/valgus and/or anteversion/ retroversion) or non-anatomic ( $>3 \mathrm{~mm}$ displacement or $>10^{\circ}$ varus/valgus and/or anteversion/retroversion) ${ }^{6,9}$ Furthermore, the position of the PFNA blade was evaluated and categorised, as previously described ${ }^{6,7}$ by dividing the femoral head into superior, central and inferior thirds on the ap radiograph and into anterior, central and posterior thirds on the lateral radiograph (Fig. 5). Cement distribution was measured in relation to the blade. Therefore, the plain X-rays in both ap and lateral view were assessed by using the software Osirix ${ }^{\mathrm{TM}}$, which allows drawing the borders of the cement and calculating the square centimetres of the marked area. The blade was subdivided in its middle in

Please cite this article in press as: Kammerlander C, et al. Standardised cement augmentation of the PFNA using a perforated blade: A new technique and preliminary clinical results. A prospective multicentre trial. Injury (2011), doi:10.1016/j.injury.2011.07.010 


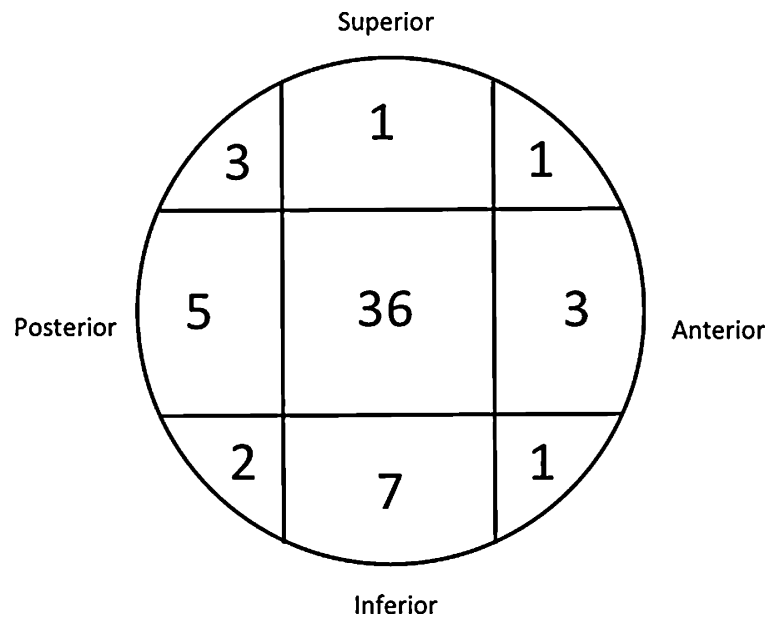

Fig. 5. The position of the blade within the femoral head.

longitudinal direction to measure the distribution of cement above and below the blade as well as centrally in the ap radiograph, in which 'centrally' means the area 'b' in Fig. 6 from the tip of the blade towards the pelvis in the longitudinal direction of the blade. Measurements in the lateral radiograph were conducted ventrally, dorsally and again centrally to the blade. The areas were calculated in relation to the area of the femoral head (Fig. 6). Furthermore, the amount of injected cement was documented. On the follow-up Xrays, signs of fracture healing were assessed. The migration of the blade within the head-neck fragment and the lateral blade migration were measured as previously described. ${ }^{35,36}$

Intra-operative complications included any unforeseen event during the augmentation, such as perforation with the guide wire into the hip joint and cement leakage. Potential postoperative complications were cutting out of the blade from the femoral head, cutting through the blade centrally, any unexpected blade migration, loosening of the blade, implant breakage, infection, additional fracture or bone-healing disturbances and any other general complication within the follow-up period.

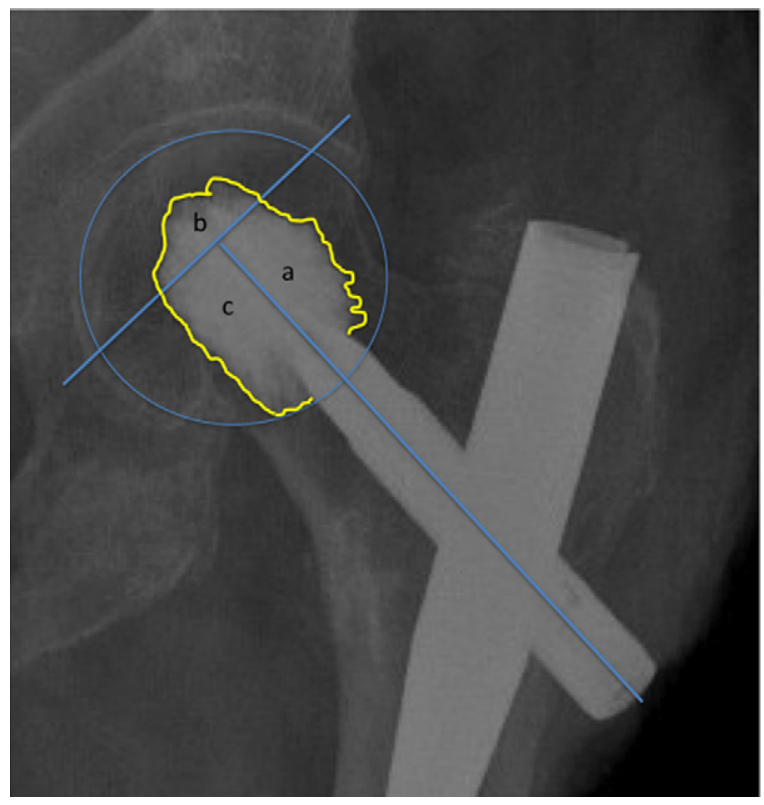

Fig. 6. The method of measuring the cement distribution around the blade in the AP view whereas a means the superior, $b$ the central and $c$ the inferior fraction of the cement.

\section{Statistical analysis}

Statistical Package for Social Sciences (SPSS) 16.0 (SPSS Inc., Chicago, IL, USA) was used for statistical analysis. All baseline and follow-up parameters were described using standard descriptive statistics. Metric scaled data are reported as arithmetic mean and categorical data as absolute frequency and percentage distribution. Depending on the distribution form, a $t$-test for independent variables or a non-parametric Mann-Whitney $U$ test was used. The Kolmogorov-Smirnov test was used to assess the distribution form. A chi-square test or a Fisher's exact test was used to analyse categorical data. The probability level was set as $p<0.05$.

\section{Results}

To investigate the effect of the standardised cement augmentation, 59 patients were analysed. Mean time to follow-up was 4 months (68-355 days). The demographics are shown in Table 1. Associated injuries were noted in two patients. The majority of the patients sustained an unstable pertrochanteric fracture (A2/3; 74.5\%). Only $20.3 \%$ were independently mobile (Parker Score 9 ) before their fracture. Mean Parker scores were 4.5 before the fracture and 3.8 at follow-up. At the follow-up, 55.3\% reached the same or even a better Parker score. Mean VAS at follow-up was 0.9. All patients were osteoporotic, whereas the mean cortical thickness index (CTI AP) was 0.47 . In $33.9 \%$, the postoperative $\mathrm{X}$-rays showed an anatomic reduction. In $57.6 \%$, reduction was near-anatomic and non-anatomic in $8.5 \%$. An open reduction was necessary in three cases, whereas in two cases subtrochanteric cerclage wires were used to stabilise the reduction without removal. In $61 \%$, the blade could be placed in an ideal position, that is, centre-centre of the head (Fig. 4). There was one patient with a postoperative bleeding and one with a rotational deformity, both necessitating a re-operation.

The only intra-operative complication reported was a perforation of the $k$-wire into the hip joint. It was detected by instilling the contrast medium (Fig. 3(b)). Consequently, no cement augmentation was performed in this patient. For the presented study, cement application was rated as "good" or "excellent" in $84.7 \%$.

In two cases, the augmented PFNA was used as a salvage procedure in failure cases. One was a failed gamma nail with lagscrew loosening in an A3 fracture. In this case, the gamma nail was removed 6 weeks after implantation and an augmented PFNA procedure was performed without complications. Follow-up examination after 4 months showed fracture union without any further problems (Fig. 7). The other case was a malreduced A3 fracture, where the PFNA was inserted at a wrong entry point and poorly positioned. In this second case, an impending cut-out was intended to be avoided with the standardised augmentation 2 weeks after the primary surgical intervention. Unfortunately, the patient died after 6 weeks due to a pre-existing renal insufficiency. Follow-up 4 weeks after the revision surgery showed no further dislocation apart from a lateral blade migration.

A mean of $4.2 \mathrm{ml}$ of cement was injected and the cement distribution around the blade was almost homogeneous (Table 2).

Table 1

The baseline characteristics of the study population.

\begin{tabular}{lll}
\hline & All $(n=59)$ & Per cent \\
\hline Age, mean & 84,5 & \\
Female/male & $45 / 14$ & $76.3 / 23.7$ \\
Left/right & $29 / 30$ & $49.2 / 50.8$ \\
AO 31-A1 & 15 & 25.4 \\
AO 31-A2 & 31 & 52.5 \\
AO 31-A3 & 13 & 22 \\
Hospitalisation time, mean & 12.5 days \\
\hline
\end{tabular}


a

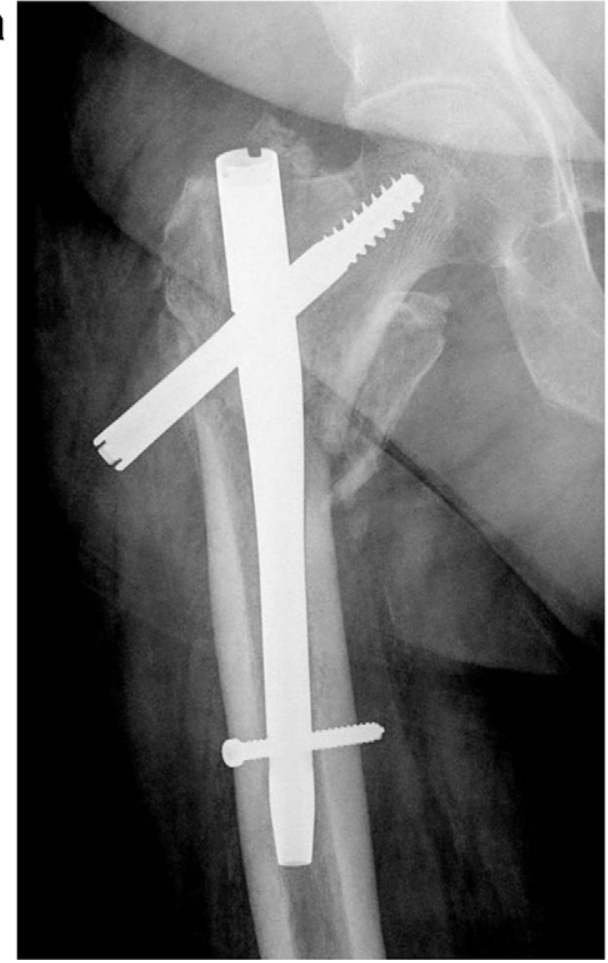

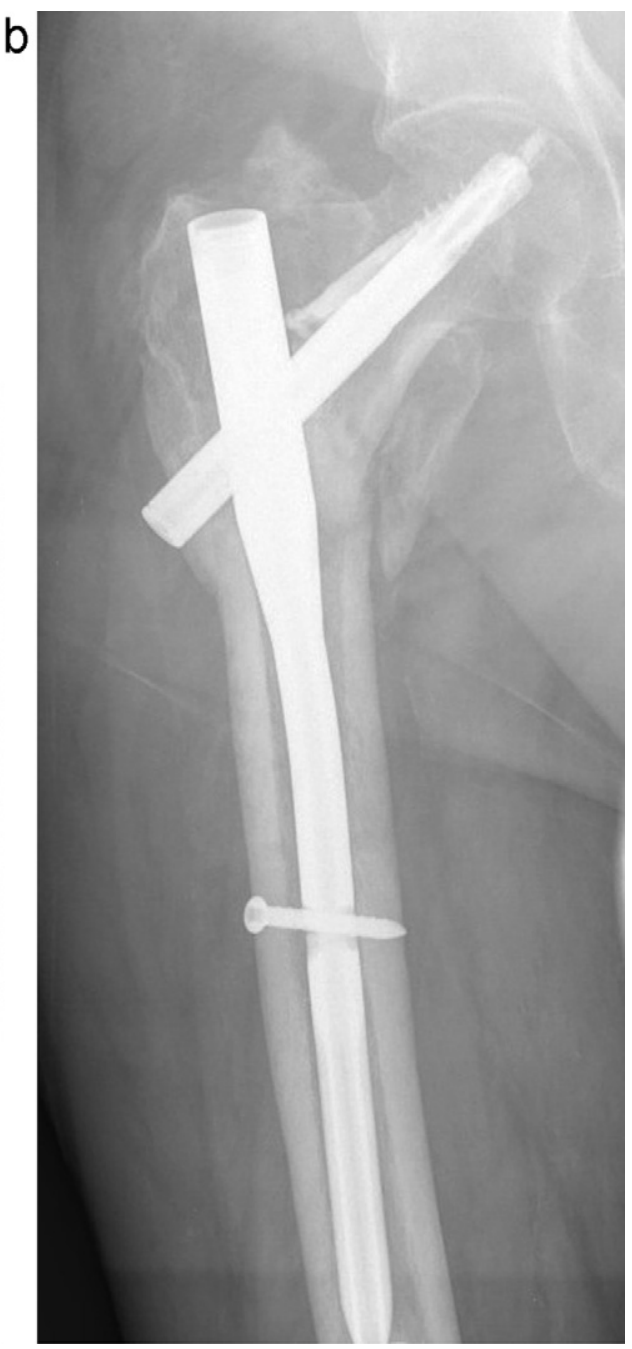

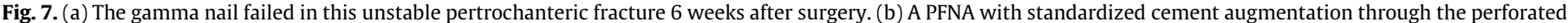
blade was used as a salvage procedure. The fracture healed without any problem within 4 months.

Only a severe osteoporosis is predictive for central (area 'b' in Fig. 6) cement flow towards the hip joint $(p<0.05)$.

At follow-up, all fractures showed a callus formation. We could not detect any sign of osteonecrosis of the femoral head or lysis around the cement. According to the above-mentioned method, ${ }^{35,36}$ there was no implant migration (e.g., migration of the blade related to the femoral head) measurable aside from lateral blade migration. We did not find any cut-out, cut through, unexpected blade migration, implant loosening or implant breakage within the study period.

Table 2

The cement distribution in all predefined directions in per cent. The right part shows the correlation ( $p$-value) with the cortical thickness index in both standard views.

\begin{tabular}{lccc}
\hline & & CTI AP & CTI AX \\
\hline $\begin{array}{l}\text { Cement distribution AP } \\
\text { Cranial }\end{array}$ & & \\
Caudal & 44.1 & 0.992 & 0.400 \\
Central & 43.1 & 0.096 & 0.061 \\
Cement distribution AX & 12.8 & 0.387 & 0.078 \\
Ventral & & & \\
Dorsal & 43.1 & 0.374 & 0.127 \\
Central & 44.8 & 0.079 & 0.089 \\
\hline
\end{tabular}

\section{Discussion}

The PFNA was proved to be a stable implant for the treatment of proximal femoral fractures. ${ }^{7-9,37}$ Nevertheless, there are complications such as cutting out of the femoral head or blade loosening reported in the literature. ${ }^{7-9}$ All these complications are catastrophic failures for these patients due to the necessity of reoperation. As most of these fractures occur in the elderly, these failures are even more severe due to the patients' co-morbidities and their inability to counterbalance, which comes along with a high perioperative morbidity and mortality.

The surgical complication rate in the presented study was $3.4 \%$, with no complication related to the cement augmentation. This is an acceptable rate compared with other reports in the literature. ${ }^{8,9}$

We did not find any cut-out or cutting through of the blade, unexpected blade migration and loosening of the blade. Implantrelated complications in proximal femoral fractures needing revision surgery, such as a cut-out of the implant through the femoral head, are reported to be as high as $16 \%^{2,10,38,39}$ Recent findings dealing only with the PFNA report about a cut-out rate of $2 \%{ }^{8}$ and $3.6 \%$. ${ }^{7}$ We did not find any cut-out or cutting through of the blade, unexpected blade migration and loosening of the blade. Therefore, our findings make us believe that an additional cement augmentation can avoid a cut-out in these fractures; but it has to be noted that our series is possibly too small to conclude this.

Please cite this article in press as: Kammerlander C, et al. Standardised cement augmentation of the PFNA using a perforated blade: A new technique and preliminary clinical results. A prospective multicentre trial. Injury (2011), doi:10.1016/j.injury.2011.07.010 
Postoperatively, we observed one case of a fall-related additional femoral fracture at the tip of the nail. In this case, the short nail was changed to a long nail and both the removal and the new implantation of the blade was performed without any problem. The blade-PMMA interface broke and there were not more force needed to remove the blade. The new blade was inserted in the same position and there was no additional augmentation done. Unfortunately, the patient died 4 weeks after the second operation due to myocardial infarction.

In our study, 55.3\% reached their prefracture functional level within the study period. In this context, we have to note that the mean age of our study population was 84.5 years and these patients have many co-morbid conditions. ${ }^{40-43}$ These co-morbidities influence the recovery of the patients ${ }^{41}$ and compared with the literature, ${ }^{7-9}$ this is a satisfying outcome.

According to cortical thickness measurements proposed by Sah et al., ${ }^{34}$ all our patients suffered from osteoporosis. We have observed that the distribution of the cement was almost homogeneous. However, it was not possible to guide the cement into a predetermined area with the used side-opening cannula. A mean amount of $4.2 \mathrm{ml}$ cement was used to augment the perforated blade. The distribution of the cement was influenced only in cases with severe osteoporosis in the way that we could observe a higher fraction central of the tip of the blade towards the hip joint. It has to be noticed that a subchondral presence of the PMMA could possibly influence the overlying cartilage. ${ }^{44}$ We did not find any correlation with the amount of injected cement and pain or mobility at follow-up. Due to an exothermic reaction during PMMA cement polymerisation, it is suspected that local bone damage may be induced. ${ }^{24}$ In our series, we did not find any radiological signs of bone necrosis. This supports the theory that there is no thermal damage to the bone due to the exothermic reaction of the PMMA in a standardised augmentation setting with only a small amount of cement injected. ${ }^{26,28}$ The mean cement volume of $4.2 \mathrm{ml}$ used in our study was able to increase stability in biomechanical investigations. ${ }^{14,20,23,26}$ However, with this small amount of cement damage to the bone is unlikely. ${ }^{14}$ In this context, it has to be mentioned that our follow-up time could be too short for a statement about bone necrosis, as several authors report the time to onset up to 3 years after the fracture. ${ }^{45,46}$ Within the study period, all fractures were healed and we did not find any bonehealing disturbance. Therefore, we state that the new standardised technique is a safe method compared with other published cement augmentation techniques. ${ }^{10,16-21,47}$

The main limitations of this study were the lack of a control group and the inclusion of stable pertrochanteric fractures. However, the feasibility of the new standardised augmentation was proved. The indication for the augmentation is not clear yet and we support the opinion that the good results with the PFNA in previous studies result from achieving a good fracture reduction and careful implant placement. ${ }^{7,8,48}$ We think that a severe osteoporosis could be a possible indication but there are probably more concomitant patient-related factors to be considered. From the socioeconomic perspective also, additional costs have to be mentioned. An analysis of failure cases and a prospective, randomised trial comparing geriatric patients with unstable pertrochanteric fractures with a PFNA either with or without augmentation would probably give us more hints to find the patients at risk and to define the indications for augmentation.

\section{Conclusion}

The standardised augmentation of the perforated PFNA blade is a safe and user-friendly tool for pertrochanteric fracture fixation. It prevents blade migration within the head-neck fragment and leads to good functional results. These impressions should be proven by a randomised trial comparing the PFNA with and without augmentation.

\section{Conflict of interest}

None of the authors has any financial or personal relationship with organisations that could influence their work inappropriately.

\section{Acknowledgements}

Synthes is acknowledged for organisational support. There was no involvement of Synthes in study planning, data analysis, interpretation or article writing. The authors would like to thank Dr. Alexander Scola for helping with data management and Dr. Stefanie Erhart for her help with preparation of the article.

\section{References}

1. Saudan M, Lubbeke A, Sadowski C, Riand N, Stern R, Hoffmeyer P. Pertrochanteric fractures: is there an advantage to an intramedullary nail? A randomized, prospective study of 206 patients comparing the dynamic hip screw and proximal femoral nail. J Orthop Trauma 2002;16(6):386-93.

2. Anglen JO, Weinstein JN. Nail or plate fixation of intertrochanteric hip fractures: changing pattern of practice. A review of the American Board of Orthopaedic Surgery Database. J Bone Joint Surg Am 2008;90(4):700-7.

3. Ahrengart L, Törnkvist H, Fornander P, Thorngren K-G, Pasanen L, Wahlström P, et al. A randomized study of the compression hip screw and Gamma nail in 426 fractures. Clin Orthop Relat Res 2002;(401):209-22.

4. Madsen JE, Naess L, Aune AK, Alho A, Ekeland A, Stromsoe K. Dynamic hip screw with trochanteric stabilizing plate in the treatment of unstable proximal femoral fractures: a comparative study with the Gamma nail and compression hip screw. J Orthop Trauma 1998;12(4):241-8.

5. Pervez H, Parker MJ, Vowler S. Prediction of fixation failure after sliding hip screw fixation. Injury 2004;35(10):994-8.

6. Vidyadhara S, Rao SK. One and two femoral neck screws with intramedullary nails for unstable trochanteric fractures of femur in the elderly - randomised clinical trial. Injury 2007;38(7):806-14.

7. Mereddy P, Kamath S, Ramakrishnan M, Malik H, Donnachie N. The AO/ASIF proximal femoral nail antirotation (PFNA): a new design for the treatment of unstable proximal femoral fractures. Injury 2009;40(4):428-32.

8. Simmermacher RK, Ljungqvist J, Bail H, Hockertz T, Vochteloo AJ, Ochs U, et al. The new proximal femoral nail antirotation (PFNA) in daily practice: results of a multicentre clinical study. Injury 2008;39(8):932-9.

9. Liu Y, Tao R, Liu F, Wang Y, Zhou Z, Cao Y, et al. Mid-term outcomes after intramedullary fixation of peritrochanteric femoral fractures using the new proximal femoral nail antirotation (PFNA). Injury 2010;41(8):810-7.

10. Mattsson $P$, Larsson $S$. Unstable trochanteric fractures augmented with calcium phosphate cement. A prospective randomized study using radiostereometry to measure fracture stability. Scand J Surg 2004;93(3):223-8.

11. Mattsson P, Larsson S. Stability of internally fixed femoral neck fractures augmented with resorbable cement. A prospective randomized study using radiostereometry. Scand J Surg 2003;92(3):215-9.

12. Bonnaire F, Weber A, Bösl O, Eckhardt C, Schwieger K, Linke B. "Cutting out" bei pertrochantären Frakturen - ein Problem der Osteoporose? Der Unfallchirurg 2007;110(5):425-32.

13. Stoffel KK, Leys T, Damen N, Nicholls RL, Kuster MS. A new technique for cement augmentation of the sliding hip screw in proximal femur fractures. Clin Biomech (Bristol Avon) 2008;23(1):45-51.

14. von der Linden P, Gisep A, Boner V, Windolf M, Appelt A, Suhm N. Biomechanical evaluation of a new augmentation method for enhanced screw fixation in osteoporotic proximal femoral fractures. J Orthop Res 2006;24(12):2230-7.

15. Szpalski M, Descamps P-Y, Hayez J-P, Raad E, Gunzburg R, Keller TS, et al. Prevention of hip lag screw cut-out by cement augmentation: description of a new technique and preliminary clinical results. J Orthop Trauma 2004;18(1): 34-40.

16. Dall'Oca C, Maluta T, Moscolo A, Lavini F, Bartolozzi P. Cement augmentation of intertrochanteric fractures stabilised with intramedullary nailing. Injury 2010;41(11):1150-5.

17. Harrington KD. The use of methylmethacrylate as an adjunct in the internal fixation of unstable comminuted intertrochanteric fractures in osteoporotic patients. J Bone Joint Surg Am 1975;57(6):744-50.

18. Schatzker J, Ha'eri GB, Chapman M. Methylmethacrylate as an adjunct in the internal fixation of intertrochanteric fractures of the femur.J Trauma 1978;18(10): 732-5.

19. Muhr G, Tscherne H, Thomas R. Comminuted trochanteric femoral fractures in geriatric patients: the results of 231 cases treated with internal fixation and acrylic cement. Clin Orthop Relat Res 1979;(138):41-4.

20. Mattsson P, Alberts A, Dahlberg G, Sohlman M, Hyldahl HC, Larsson S. Resorbable cement for the augmentation of internally-fixed unstable trochanteric fractures. A prospective, randomised multicentre study. J Bone Joint Surg $\mathrm{Br}$ 2005;87(9):1203-9. 
21. Bartucci EJ, Gonzalez MH, Cooperman DR, Freedberg HI, Barmada R, Laros GS. The effect of adjunctive methylmethacrylate on failures of fixation and function in patients with intertrochanteric fractures and osteoporosis. J Bone Joint Surg Am 1985;67(7):1094-107.

22. Kammerlander C, Blauth M, Roth T. Re: cement augmentation of intertrochanteric fractures stabilised with intramedullary, in press.

23. Lindner T, Kanakaris NK, Marx B, Cockbain A, Kontakis G, Giannoudis PV. Fractures of the hip and osteoporosis: the role of bone substitutes. J Bone Joint Surg Br 2009;91(3):294-303.

24. Heini PF, Franz T, Fankhauser C, Gasser B, Ganz R. Femoroplasty-augmentation of mechanical properties in the osteoporotic proximal femur: a biomechanical investigation of PMMA reinforcement in cadaver bones. Clin Biomech (Bristo Avon) 2004;19(5):506-12.

25. von Steyern FV, Kristiansson I, Jonsson K, Mannfolk P, Heinegard D, Rydholm A Giant-cell tumour of the knee: the condition of the cartilage after treatment by curettage and cementing. J Bone Joint Surg Br 2007;89(3):361-5.

26. Boner V, Kuhn P, Mendel T, Gisep A. Temperature evaluation during PMMA screw augmentation in osteoporotic bone - an in vitro study about the risk of thermal necrosis in human femoral heads. J Biomed Mater Res B Appl Biomater 2009;90(2):842-8.

27. Unsal M, Tetik C, Erol B, Cabukoglu C. The injection of acrylic bone cement prevents bone collapse in the intercalar bones lacking bony support: an experimental sheep semilunar bone model. Acta Orthop Traumatol Turc 2003;37(1):63-9.

28. Welch RD, Berry BH, Crawford K, Zhang H, Zobitz M, Bronson D, et al. Subchondral defects in caprine femora augmented with in situ setting hydroxyapatite cement, polymethylmethacrylate, or autogenous bone graft: biomechanical and histomorphological analysis after two-years. J Orthop Res 2002;20(3):464-72.

29. Oken MM, Creech RH, Tormey DC, Horton J, Davis TE, McFadden ET, et al. Toxicity and response criteria of the Eastern Cooperative Oncology Group. Am J Clin Oncol 1982;5(6):649-55.

30. Parker MJ, Palmer CR. A new mobility score for predicting mortality after hip fracture. J Bone Joint Surg Br 1993;75(5):797-8.

31. Carlsson AM. Assessment of chronic pain. I. Aspects of the reliability and validity of the visual analogue scale. Pain 1983;16(1):87-101.

32. Bryant DM, Sanders DW, Coles CP, Petrisor BA, Jeray KJ, Laflamme GY. Selection of outcome measures for patients with hip fracture. J Orthop Trauma 2009;23(6):434-41.

33. Dorr LD, Faugere MC, Mackel AM, Gruen TA, Bognar B, Malluche HH. Structural and cellular assessment of bone quality of proximal femur. Bone 1993;14(3): 231-42.
34. Sah AP, Thornhill TS, Leboff MS, Glowacki J. Correlation of plain radiographic indices of the hip with quantitative bone mineral density. Osteoporos Int 2007;18(8):1119-26.

35. Watanabe Y, Minami G, Takeshita H, Fujii T, Takai S, Hirasawa Y. Migration of the lag screw within the femoral head: a comparison of the intramedullary hip screw and the Gamma Asia-Pacific nail. J Orthop Trauma 2002;16(2):104-7.

36. Gardner MJ, Briggs SM, Kopjar B, Helfet DL, Lorich DG. Radiographic outcomes of intertrochanteric hip fractures treated with the trochanteric fixation nail. Injury 2007;38(10):1189-96.

37. Lenich A, Vester H, Nerlich M, Mayr E, Stockle U, Fuchtmeier B. Clinical comparison of the second and third generation of intramedullary devices for trochanteric fractures of the hip - blade vs screw. Injury 2010;41(12):1292-6.

38. Parker MJ. Valgus reduction of trochanteric fractures. Injury 1993;24(5):313-6.

39. Simpson AH, Varty K, Dodd CA. Sliding hip screws: modes of failure. Injury 1989;20(4):227-31.

40. Donegan DJ, Gay AN, Baldwin K, Morales EE, Esterhai Jr JL, Mehta S. Use of medical comorbidities to predict complications after hip fracture surgery in the elderly. J Bone Joint Surg Am 2010;92(4):807-13.

41. Roche JJ, Wenn RT, Sahota O, Moran CG. Effect of comorbidities and postoperative complications on mortality after hip fracture in elderly people: prospective observational cohort study. BMJ 2005;331(7529):1374.

42. Kammerlander C, Roth T, Friedman SM, Suhm N, Luger TJ, KammerlanderKnauer U, et al. Ortho-geriatric service - a literature review comparing different models. Osteoporos Int 2010;21(Suppl. 4):S637-46.

43. Roth T, Kammerlander C, Gosch M, Luger TJ, Blauth M. Outcome in geriatric fracture patients and how it can be improved. Osteoporos Int 2010;21(Suppl. 4):S615-9.

44. Hisatome T, Yasunaga Y, Ikuta Y, Fujimoto Y. Effects on articular cartilage of subchondral replacement with polymethylmethacrylate and calcium phosphate cement. J Biomed Mater Res 2002;59(3):490-8.

45. Barnes R, Brown JT, Garden RS, Nicoll EA. Subcapital fractures of the femur. A prospective review. J Bone Joint Surg Br 1976;58(1):2-24.

46. Loizou CL, Parker MJ. A vascular necrosis after internal fixation of intracapsular hip fractures; a study of the outcome for 1023 patients. Injury 2009;40(11): 1143-6.

47. Szpalski M, Descamps PY, Hayez JP, Raad E, Gunzburg R, Keller TS, et al. Prevention of hip lag screw cut-out by cement augmentation: description of a new technique and preliminary clinical results. J Orthop Trauma 2004;18(1): 34-40.

48. Lobo-Escolar A, Joven E, Iglesias D, Herrera A. Predictive factors for cutting-out in femoral intramedullary nailing. Injury 2010;41(12):1312-6. 